TRANSACTIONS OF THE

AMERICAN MATHEMATICAL SOCIETY

Volume 361, Number 7, July 2009, Pages 3611-3632

S 0002-9947(09)04659-5

Article electronically published on February 4, 2009

\title{
FROBENIUS-SCHUR INDICATORS FOR SUBGROUPS AND THE DRINFEL'D DOUBLE OF WEYL GROUPS
}

\author{
ROBERT GURALNICK AND SUSAN MONTGOMERY
}

\begin{abstract}
If $G$ is any finite group and $k$ is a field, there is a natural construction of a Hopf algebra over $k$ associated to $G$, the Drinfel'd double $D(G)$. We prove that if $G$ is any finite real reflection group, with Drinfel'd double $D(G)$ over an algebraically closed field $k$ of characteristic not 2 , then every simple $D(G)$-module has Frobenius-Schur indicator +1 . This generalizes the classical results for modules over the group itself. We also prove some new results about Weyl groups. In particular, we prove that any abelian subgroup is inverted by some involution. Also, if $E$ is any elementary abelian 2-subgroup of the Weyl group $W$, then all representations of $C_{W}(E)$ are defined over $\mathbb{Q}$.
\end{abstract}

\section{INTRODUCTION}

There has been considerable interest lately in using Frobenius-Schur indicators in studying semisimple Hopf algebras, as they are one of only a few useful invariants for a Hopf algebra $H$. For example indicators (and their higher analogs) have been used in classifying Hopf algebras themselves [13] [23], in studying possible dimensions of the representations of $H$ [15, and in studying the prime divisors of the exponent of $H$ [16]. Moreover, the indicator is invariant under equivalence of monoidal categories [20. Another motivation comes from conformal field theory; see works of Bantay [1, 2]. Thus it is important to compute the values of the indicator for more examples.

Let $k$ be an algebraically closed field of characteristic not 2, $H$ an involutive Hopf algebra (i.e. the antipode has order 2) and $V$ a finite-dimensional irreducible $H$-module. We show (generalizing the case of group algebras and semisimple Hopf algebras) that one can define the Schur indicator of $V$ and it has the following properties: $\nu(V) \neq 0$ if and only if $V$ is self dual, $\nu(V)=+1$ if and only if $V$ admits a non-degenerate $H$-invariant symmetric bilinear form and $\nu(V)=-1$ if and only $V$ admits a non-degenerate $H$-invariant skew-symmetric bilinear form. If $G$ is a finite group, then self duality is equivalent to $\chi(x)=\chi\left(x^{-1}\right)$ for all $x \in G$ where $\chi$ is the character of $V$. In particular, if $k=\mathbb{C}$, this is saying that the character is real valued (and all representations are self dual if and only if $x$ and $x^{-1}$ are conjugate in $G$ for all $\left.x\right)$. Also, $\nu(V)=+1$ if and only if $V$ is defined over $\mathbb{R}$.

Received by the editors March 26, 2007.

2000 Mathematics Subject Classification. Primary 16W30, 20C15, 20G42.

Key words and phrases. Drinfel'd double, Schur indicator, Weyl groups, reflection groups, rationality of representations.

The authors were supported by NSF grants DMS 0140578 and DMS 0401399.

(C)2009 American Mathematical Society 
It was shown in [18] that the classical theorem of Frobenius and Schur, giving a formula to compute the indicator of a simple module for a finite group $G$, extends to semisimple Hopf algebras. This fact was shown a bit earlier for the special case of Kac algebras over $\mathbb{C}$ in [6. In this paper, we show that the indicator is well defined whenever the antipode of $H$ satisfies $S^{2}=$ id. Moreover, the indicator has the above characterization in terms of bilinear forms. The formula for the indicator is no longer valid (this is clear already for modular group algebras).

The case when $\nu(V)=+1$ for all simple $G$-modules $V$ has been of particular interest; such groups are called totally orthogonal in [10. This terminology seems suitable for Hopf algebras as well, in view of the existence of the bilinear forms described above. In particular it was known classically that if $G$ is any finite real reflection group, then all $\nu(V)=+1$ [11]; other examples are given in [10].

For Hopf algebras, there has also been some interest in when a Hopf algebra is "totally orthogonal", in particular when $H=D(G)$, the Drinfel'd double of a finite group. It is proved in [14 that if $G$ is a dihedral group or the symmetric group $\operatorname{Sym}_{n}$ and $k=\mathbb{C}$, then $D(G)$ is totally orthogonal. More generally, 14] studies the indicator for Hopf algebras which are abelian extensions, since in this case the irreducible representations are known.

A natural question to ask is what happens for the other Weyl groups, since their group algebras in any characteristic not 2 have all indicators for irreducible modules equal to +1 . The main result we prove for Drinfel'd doubles is:

Theorem 1.1. Let $G$ be a finite real reflection group and $D(G)$ its Drinfel'd double over the algebraically closed field $k$ of characteristic not 2 . Then $D(G)$ is totally orthogonal; that is, every simple $D(G)$-module has Frobenius-Schur indicator +1 .

Recall that a real reflection group is a subgroup of $G L(V)$ with $V$ finite dimensional over $\mathbb{R}$ that is generated by reflections. Every such real reflection group is a direct product of irreducible real reflection groups. The finite irreducible real reflection groups are well known (cf. [11]); aside from some examples in dimension at most 4, they are the Weyl groups, and for dimension greater than 8 , they are just the classical Weyl groups.

We also prove some new general results for real reflection groups and Weyl groups which give more than just our main theorem and are of independent interest. We prove these results by considering first the classical Weyl groups (that is, those of types A, B and D). For the smaller reflection groups, we use computer calculations - some of which were quite simple and some more involved. We refer the reader to [3] and 7] for more details.

We should point out that it is false in general that if $G$ is totally orthogonal, then the same is true for $D(G)$. We give an example of a group of order 32 with the property that all of its representations are defined over $\mathbb{Q}$ but such that $D(G)$ has an irreducible module with indicator 0 (see Section 9). Thus a proof of Theorem 1.1 will have to involve the specific properties of the reflection groups. Indeed, we shall see that the classical Weyl groups satisfy some stronger properties than all real reflection groups (or even all Weyl groups).

We now discuss some of our results about Weyl groups and real reflection groups. If $g \in G$, let $C_{g}$ denote the centralizer of $g$ and $N_{g}$ the normalizer of $\left\{g, g^{-1}\right\}$.

Theorem 1.2. Let $G$ be a finite real reflection group and let $A$ be an abelian subgroup of $G$. 
(1) There exists an involution $t \in G$ that acts as inversion on $A$.

(2) If $G$ is a Weyl group and $r$ is relatively prime to $|A|$, then there exists $x \in G$ with $x a x^{-1}=a^{r}$ for all $a \in A$.

The first part of the theorem is a generalization of the well known property for Weyl groups that every element is a product of two involutions (cf. [28, §8]). This also implies that all representations of $C_{g}$ are self dual, but we show more:

Theorem 1.3. Let $G$ be a real reflection group. Let $E$ be an elementary abelian 2 -subgroup of $G$ and let $k$ be an algebraically closed field of characteristic $p \neq 2$.

(1) Every element of $C_{G}(E)$ is a product of 2 involutions, and $k C_{G}(E)$ is totally orthogonal; and

(2) If $G$ is a Weyl group, then every irreducible complex representation of $C_{G}(E)$ is defined over $\mathbb{Q}$.

Let $L$ be a field. Recall that the field of definition for a module $V$ for $L G$ is the smallest field $K$ such that $V=U \otimes_{K} L$ with $U$ a $K G$-module (of course, $K$ must contain all character values, and in positive characteristic this is sufficient as well for $V$ semisimple).

We prove the second assertion in Theorem 1.3 of this paper for the classical Weyl groups. It is proved for the exceptional Weyl groups in 7]. In particular, this generalizes the well known result that all representations of Weyl groups are defined over $\mathbb{Q}$. Our proof is independent of this (we do use the relatively elementary result for symmetric groups).

We also prove:

Theorem 1.4. Let $G$ be a real reflection group and $k$ an algebraically closed field of characteristic $p \neq 2$. Let $g \in G$.

(1) If $G$ is a classical Weyl group or is a real reflection group of dimension at most 3, then all Frobenius-Schur indicators for irreducible $k N_{g}$-modules are +1 .

(2) For all other real reflection groups, all Frobenius-Schur indicators for irreducible $k N_{g}$-modules are non-negative.

One computes easily that for the Weyl groups of type $E$ and $F$ and for the real reflection group $H_{4}$, there exists an element of order 3 such that $N_{g}$ is not totally orthogonal. Thus, one cannot expect a uniform proof.

We will show that the two previous theorems are enough to show that $D(G)$ is totally orthogonal for $G$ a finite real reflection group. A curious consequence of the result on $D(G)$ gives a formula for the finite reflection groups that seems new:

Corollary 1.5. Let $G$ be a finite reflection group. Let $t$ be the number of elements of $G$ whose square is trivial. Then

$$
t^{2}=\sum_{g \in G} \sum_{\chi \in \operatorname{Irr}\left(C_{g}\right)} \chi(1)
$$

Indeed, this formula holds for a finite group if and only if $D(G)$ is totally orthogonal. This follows since $t^{2}$ is the trace of the antipode of $D(G)$ and when all indicators are +1 , this is equal to the right hand side (see [18], [14]). 
The paper is organized as follows. In Section 2 we review and extend the results from 14] which we need, and give a refinement of the criterion for positivity of Schur indicators in 14. We also generalize the result to any characteristic other than 2 and do not assume that $D(G)$ is semisimple. In order to do this, we first show that the indicator is well defined when $H$ is not necessarily semisimple as long as $S^{2}=$ id. In Section 3, we review some basic properties of Schur indices of finite groups.

In Section 4 we consider the case of direct products and we also show that it is sufficient to prove Theorem 1.1 in characteristic 0. In Section [5] we consider normalizers of abelian subgroups of Weyl groups. In particular, we prove Theorem 1.2 and the results about centralizers of involutions. In Section 6, we show that certain groups are split over $\mathbb{R}$. In Section 7 we consider the classical Weyl groups. In Section 8 we consider the remaining real reflection groups. For these small cases, we obtain the results by computation (some quite easy and some more involved). We also prove Corollary 1.5. Finally in Section 9 we discuss the example showing that total orthogonality does not always lift from $G$ to $D(G)$.

\section{Indicators For involutive Hopf Algebras AND Drinfel'D doubles}

We recall a few basic facts about modules over a Hopf algebra $H$ over a field $k$. We assume throughout that $S^{2}=\mathrm{id}$, for the antipode $S$ of $H$. This is always the case by [17] for semisimple Hopf algebras in characteristic zero and so is also the case for Hopf algebras in positive characteristic which may be defined by reducing semisimple Hopf algebras modulo $p$ (e.g., group algebras, Drinfe'ld doubles of finite groups and bismash products of groups). Masuoka has pointed out to us that it is also true for bicrossed products of groups, using descent arguments as in [21].

Let $\mathcal{C}={ }_{H} \mathcal{M}$ denote the category of finite-dimensional left $H$-modules. Let $V_{0}=k v_{0} \in \mathcal{C}$ denote the trivial module; that is $h \cdot v_{0}=\varepsilon(h) v_{0}$. If $V, W \in \mathcal{C}$, then $V \otimes W \in \mathcal{C}$, via $h \cdot(v \otimes w):=\Delta(h) \cdot(v \otimes w)=\sum h_{1} \cdot v \otimes h_{2} \cdot w$, for all $h \in H, v \in$ $V, w \in W$, and also $\operatorname{Hom}_{k}(V, W) \in \mathcal{C}$ via $(h \cdot f)(v):=\sum h_{1} \cdot\left(f\left(S h_{2} \cdot v\right)\right) \in W$. As a special case, the dual module $V^{*} \in \mathcal{C}$ also, via $(h \cdot f)(v)=f(S h \cdot v)$ for all $h \in H, f \in H^{*}$ and $v \in V$. Since $S^{2}=i d, V^{* *} \cong V$.

For any $V \in \mathcal{C}$, let $V^{H}:=\{v \in V \mid h \cdot v=\varepsilon(h) v \forall h \in H\}$, the $H$-invariants of $V$.

In [18, the indicator was defined for semisimple Hopf algebras in terms of a formula involving integrals. This definition does not work in the non-semisimple case, since in that case there does not exist an integral $\Lambda \in \int_{H}$ with $\epsilon(\Lambda)=1$. Instead we define the indicator in terms of the existence (or non-existence) of certain invariant bilinear forms, which is the conclusion of [18, Theorem 3.1, (1) and (2)]. This definition agrees with the classical definition of an indicator for finite groups in characteristic $p$; see for example [29, p. 227].

More generally, when $S^{2}$ is given by conjugation by a group-like element, the representation category is pivotal. Recently, in [25], there is a definition of indicator in that context, in terms of the trace of a certain map, but not in terms of invariant bilinear forms.

Definition 2.1. Let $H$ be a Hopf algebra over an algebraically closed field of characteristic not 2 such that $S^{2}=$ id, and let $V$ be a finite-dimensional irreducible 
(left) $H$-module. We define a function $\nu: V \rightarrow\{0,1,-1\}$, called the FrobeniusSchur indicator of $V$, as follows:

(1) If $V$ is not self dual, set $\nu(V)=0$.

(2) If $V^{*} \cong V$ and $V$ admits a non-degenerate $H$-invariant symmetric bilinear form, set $\nu(V)=+1$.

(3) If $V^{*} \cong V$ and $V$ admits a non-degenerate $H$-invariant skew-symmetric form, set $\nu(V)=-1$.

We prove here that in fact any irreducible $H$-module $V$ is of precisely types (1), (2), or (3). We first need an elementary lemma, most of which is well known.

Lemma 2.2. Let $V, W \in \mathcal{C}$. Then as left $H$-modules,

(1) $\operatorname{Hom}\left(V, W^{*}\right) \cong \operatorname{Hom}(V \otimes W, k)$ and $\operatorname{Hom}_{H}(V, W)=\operatorname{Hom}(V, W)^{H}$.

(2) Assume $k$ is algebraically closed and $V$ is irreducible. If $V^{*} \cong V$, then $\operatorname{Hom}_{H}(V \otimes V, k)$ is one-dimensional, and if $V^{*} \nsucceq V$, then $\operatorname{Hom}_{H}(V \otimes V, k)=$ 0 .

(3) Assume $k$ is algebraically closed, $V$ is irreducible, $V^{*} \cong V$, and choose $0 \neq\langle-,-\rangle \in \operatorname{Hom}_{H}(V \otimes V, k)=\left((V \otimes V)^{*}\right)^{H}$. Then

$$
\sum\left\langle h_{1} \cdot v, h_{2} \cdot w\right\rangle=\varepsilon(h)\langle v, w\rangle .
$$

(4) The form $\langle-,-\rangle \in \operatorname{Hom}_{H}(V \otimes V, k) \Longleftrightarrow$ the antipode $S$ is the adjoint with respect to the form.

Proof. (1) The first part is straightforward, using the standard map $\operatorname{Hom}\left(V, W^{*}\right) \rightarrow$ $\operatorname{Hom}(V \otimes W, k)$ given by $f \mapsto \tilde{f}$, where $\tilde{f}(v \otimes w):=f(v)(w)$. The second fact follows from the definitions.

(2) follows from (1) using $W=V$ and Schur's Lemma.

(3) The displayed equation is simply saying that $\langle-,-\rangle$ is an $H$-module map. The fact that the form may be considered as an invariant of $(V \otimes V)^{*}$ follows from (1).

(4) Finally, we consider the relationship of the form $\langle-,-\rangle$ to $S$. This is an old fact; see for example [18, [16]. Now $\langle-,-\rangle \in \operatorname{Hom}_{H}(V \otimes V, k)$ means that for all $h \in H, v, w \in V, \sum\left\langle h_{1} \cdot v, h_{2} \cdot w\right\rangle=\varepsilon(h)\langle v, w\rangle$; that is, $\langle-,-\rangle$ is $H$-invariant. To say that $S$ is the adjoint of the form means that $\langle h \cdot v, w\rangle=\langle v, S h \cdot w\rangle$ for all $h, v, w$.

The equivalence of these two conditions follows easily from the counit property of $\varepsilon$ and from the fact that $S$ is a "generalized inverse" map on $H$; that is, for any $h \in H$ with $\Delta(h)=\sum h_{1} \otimes h_{2}$, both $\sum \varepsilon\left(h_{1}\right) h_{2}=h$ and $S\left(h_{1}\right) h_{2}=\varepsilon(h)$.

We note that a more general version of the equivalence in (4) is shown in 20] for quasi-Hopf algebras.

From (2), every $H$-invariant form on $V \cong V^{*}$ is a scalar multiple of the given one $0 \neq\langle-,-\rangle$. Given a form $\langle-,-\rangle$ on $V \cong V^{*}$, we may also consider its "twist" $\tau$; that is, the form $[v, w]:=\tau(\langle v, w\rangle)=\langle w, v\rangle$.

The argument in the next lemma is taken from [16, p. 18]. Their assumption that $H$ is semisimple is not used in the proof; it only used the fact that $S^{2}=$ id. 
Lemma 2.3. If $\langle-,-\rangle$ is $H$-invariant, then so is its twist $[v, w]=\langle w, v\rangle$. Consequently $\langle w, v\rangle=\alpha\langle w, v\rangle$, for all $v, w$, where $\alpha= \pm 1$ is independent of the choice of $\langle-,-\rangle$ and depends only on $V$.

Proof. Using $S^{2}=$ id and Lemma 2.2,

$$
[h \cdot v, w]=\left[S^{2}(h) \cdot v, w\right]=\left\langle w, S^{2}(h) \cdot v\right\rangle=\langle S(h) \cdot w, v\rangle=[v, S(h) \cdot w],
$$

for any $v, w \in V, h \in H$.

Since $\left((V \otimes V)^{*}\right)^{H}$ is one dimensional, $[-,-]=\alpha\langle-,-\rangle$ for some $\alpha \in k$. Repeating the argument gives $\alpha^{2}=1$, and so $\alpha= \pm 1$. It follows that $\alpha$ is independent of the choice of $\langle-,-\rangle$ and depends only on $V$.

The theorem is now clear from our arguments above.

Theorem 2.4. Let $H$ be a Hopf algebra such that $S^{2}=i d$, over the algebraically closed field $k$ of characteristic $p \neq 2$, and let $V$ be a finite-dimensional irreducible (left) $H$-module. Then $V$ falls into case (1), (2), or (3) of the definition, and so has a Frobenius-Schur indicator. Moreover,

(1) $V^{*} \cong V \Longleftrightarrow \nu(V) \neq 0 \Longleftrightarrow(V \otimes V)^{H}$ is one-dimensional; and

(2) if $\nu(V) \neq 0$ and $f$ is a non-zero invariant in $V \otimes V$, then $\tau(f)=\nu(V) f$, where $\tau$ is the twist map above.

We now consider Drinfel'd doubles. Throughout the remainder of the section, let $k$ be an algebraically closed field of characteristic $p$ with $p \neq 2$.

For a finite group $G$, let $k G$ be the group algebra and $(k G)^{*}$ its dual Hopf algebra, with dual bases $\{g \in G\}$ and $\left\{p_{g} \mid g \in G\right\}$. The Drinfel'd double $D(G)$ of $G$ is simply $(k G)^{*} \otimes k G$ as a vector space. As an algebra it is the smash product $(k G)^{*} \# k G$, where $k G$ acts on $(k G)^{*}$ via $g \cdot p_{h}=p_{g h g^{-1}}$, for all $g, h \in G$. The coalgebra structure is given by the tensor product of coalgebras, and the antipode $S$ is given by

$$
S\left(p_{x} \# a\right)=p_{a x^{-1} a^{-1}} \# a^{-1} .
$$

One sees directly that $S^{2}=$ id.

The description of the simple $D(G)$-modules is well known [4, [19, Section 2], and in fact depends only on the algebra structure of $D(G)$. More precisely, for any $g \in G$ let $C_{g}$ denote the centralizer of $g$ in $G$, and choose one $g$ in each conjugacy class of $G$. For each irreducible $C_{g}$-module $V$, let $\hat{V}=V_{C_{g}}^{G}$ be the induced module; then $\hat{V}$ is a $k G$-module, and it becomes a $D(G)$-module via

$$
\left(p_{h} \# y\right) \cdot[x \otimes v]=\delta_{x g x^{-1}, h}[y x \otimes v]
$$

for $h, x \in G$ and $v \in V$.

Then $\hat{V}$ is an irreducible $D(G)$-module, and all irreducible $D(G)$-modules arise in this way. Thus the simple $D(G)$-modules $\hat{V}$ are indexed by pairs $(g, V)$, where $g \in G$ ranges over a set of conjugacy class representatives and $V$ is an irreducible $C_{g}$-module.

We want to refine the result [14, Theorem 5.5] about Schur indicators of the simple $H$-modules for $H=D(G)$. We also extend the results to the case of positive characteristic without the assumption that $D(G)$ is semisimple. Thus, our proof will be both more general and independent of [14].

As in [14, we will show that to compute $\nu(\hat{V})$ one does not need to induce $V$ up to $G$, but only up to $N_{g}$, the normalizer of the set $\left\{g, g^{-1}\right\}$ in $G$, and then use the usual indicator for the representations of the groups $C_{g}$ and $N_{g}$. 
Theorem 2.6. Let $k$ be an algebraically closed field of characteristic not $2, G$ be a finite group, $D(G)$ be the Drinfel'd double of $G$ over $k$ and $\hat{V}$ be an irreducible $D(G)$-module corresponding to the irreducible $C_{g}$-module $V$.

(1) If $g^{2}=1$, then $\nu(\hat{V})=\nu(V)$.

(2) If $g^{2} \neq 1$, then $\nu(\hat{V})=\nu\left(V_{C_{g}}^{N_{g}}\right)-\nu(V)$.

We will prove this below in a series of results which give a bit more information.

For $V$ a $C_{g}$-module, we will denote the conjugate module to $V$ by $V^{x}$; that is, $V^{x}=V$ as a $k$-space, but for any $y \in C_{g}$ and $v \in V^{x}, y \cdot v=x y x^{-1} \cdot v$.

If $h \in G$, let $I(h)$ be the $k$-span of $\left\{p_{x} \# y \mid y \in G, x \in h^{G}\right\}$. Then $I(h)$ is a 2-sided ideal of $H$ and $H$ is the direct sum of $I(h)$ as $h$ ranges over a set of conjugacy class representatives. Moreover, $I(1) \cong k G$. Since $\varepsilon(x)=0$ for $x \in I(h), h \neq 1$, we see that the $D(G)$ invariants $W^{D(G)}$ on a module $W$ are precisely the $G$-fixed points on $I(1) W$.

We first observe:

Lemma 2.7. Let $g \in G$ and $V$ be an irreducible $k C_{g}$-module. If $g$ and $g^{-1}$ are not conjugate, then $\nu(\hat{V})=0$.

Proof. We note that $I(h) \hat{V}=0$ if and only if $h$ is not conjugate to $g$, while $I(h) \hat{V}^{*}=$ 0 if and only if $h$ is not conjugate to $g^{-1}$. So if $g$ and $g^{-1}$ are not conjugate, then clearly $\hat{V}$ is not self dual.

Theorem 2.8. Let $g \in G$ and $V$ be an irreducible $k C_{g}$-module. Set $C=C_{g}$ and $N=N_{g}$. Let $x \in G$ with $x g x^{-1}=g^{-1}$.

(1) $\nu(\hat{V}) \neq 0$ if and only if $V^{*} \cong V^{x}$ as $C_{g}$-modules.

(2) If $g^{2}=1$, then $\nu(\hat{V})=\nu(V)$.

(3) If $g^{2} \neq 1$, then $\nu(\hat{V})=\nu\left(V_{C}^{N}\right)-\nu(V)$.

Proof. We may assume that $x=1$ if $g^{2}=1$. Note that $x \in N$.

Let $S$ be a set of left coset representatives for $N$ in $G$. Then $T:=S \cup S x$ is a set of left coset representatives for $C$ in $G$. So $\hat{V}=\sum_{t \in T} t \otimes V$. Set $V_{t}:=t \otimes V$.

Set $X=\hat{V} \otimes \hat{V}$ and let $\tau$ be the natural involution on $X$.

It follows easily from the definitions that $Y:=I(1) X=\sum_{t \in T} V_{t} \otimes V_{t x}$. Thus the $D(G)$-invariants on $X$ are precisely the $G$-fixed points on $Y$. Note that $Y \cong$ $\left(V \otimes V_{x}\right)_{C}^{G}$. By Frobenius reciprocity, $G$ has fixed points on $Y$ if and only if $C$ has fixed points on $V \otimes V_{x}$. Since $V_{x} \cong V^{x}$ as $k C$-modules, we see that $D(G)$ has invariants on $X$ if and only if $V \cong V^{*}$ as $C$-modules. This proves (1).

Note that Frobenius reciprocity gives a bit more. It tells us that if $f$ is a non-zero $C$-fixed point on $V \otimes V_{x}$, then $F:=\sum_{t \in T} t f$ is a non-zero fixed point of $G$ on $Y$ (and so a non-zero $D(G)$-invariant).

Suppose that $g^{2}=1$. Then $V \otimes V$ is $\tau$-invariant and $\tau$ commutes with the action of $C$ on $V \otimes V$. So if $f$ is a non-zero $C$-fixed element, then $\tau(f)=\nu(V) f$ and $\tau(F)=\nu(V) F$, whence $\nu(V)=\nu(\hat{V})$. This proves (2).

Now assume that $g^{2} \neq 1$. If $V^{x}$ and $V^{*}$ are non-isomorphic $C$-modules, then it is straightforward to compute that $\nu(V)=\nu\left(V_{C}^{N}\right)$, and so (3) holds in this case.

So finally, we assume also that $V^{x}$ and $V^{*}$ are isomorphic as $C$-modules.

Let $U=\left(V \otimes V_{x}\right) \oplus\left(V_{x} \otimes V\right)$. Note that $U$ is an $N$-module and is $\tau$-invariant. Also, it is clear that $Y \cong U_{N}^{G}$. Thus, arguing as above, we deduce that there is 
a non-zero $N$-fixed point $f$ in $U$ and $\tau(f)=\nu(\hat{V}) f$. We have now reduced the computation of $\nu(\hat{V})$ to a computation in $N$, and we proceed to prove (3).

Let $W=V_{C}^{N}$ and note that $U$ embeds in $W \otimes W$. Also, the action of $\tau$ on $U$ extends to that of $W \otimes W$ in the obvious manner (with $\tau$ the natural involution on $W \otimes W$ ). The result now follows by the group theoretic result, Lemma 2.9 below.

Lemma 2.9. Let $k$ be an algebraically closed field of characteristic $p \neq 2$. Let $C$ be a subgroup of index 2 in the finite group $N$. Fix an element $x \in N \backslash C$ and let $V$ be an irreducible $k C$-module with $V^{*} \cong V^{x}$. Set $W=V_{C}^{N}=V \oplus x V$. Let $X=W \otimes W$ and let $\tau$ be the linear map sending $w_{1} \otimes w_{2}$ to $w_{2} \otimes w_{1}$. Let $Y=(V \otimes x V) \oplus(x V \otimes V)$, a $k N$-submodule of $X$. The fixed points of $N$ on $Y$ are 1-dimensional and are $\tau$-invariant. Let $f$ be a generator for this space. Then $\tau(f)=(\nu(W)-\nu(V)) f$, where $\nu(W)$ is the Frobenius-Schur indicator of $W$ as a $k N$-module and $\nu(V)$ is the Frobenius-Schur indicator of $V$ as a $k C$-module.

Proof. Note that $X=Y \oplus Z$, where $Z=V \otimes V \oplus x V \otimes x V$. Clearly $Y$ and $Z$ are both invariant under $N$ and $\tau$. Note that $C$ has a 1-dimensional fixed space on $V \otimes x V \cong V \otimes V^{*}$. Let $w$ be a generator for this space. Then $w, x w$ is a basis for the fixed space of $C$ on $Y$, and so $f:=w+x w$ is a basis for the fixed space of $N$ on $Y$. Since $\tau$ commutes with $N$ on $X, \tau f=\gamma f$ where $\gamma= \pm 1$. So we only need show that $\gamma=\nu(W)-\nu(V)$.

We consider various cases.

Case 1. $V$ is not self dual as a $k C$-module.

Then $W$ is an irreducible self dual $k N$-module, whence the $N$-invariants on $W \otimes W$ are 1-dimensional and so generated by $f$. Thus, $\gamma=\nu(W)$, and since $\nu(V)=0$, the result holds.

Case 2. $V$ is self dual. By Frobenius reciprocity, $W=U_{1} \oplus U_{2}$, where the $U_{i}$ are non-isomorphic $k N$-modules such that $U_{i} \cong V$ as $k C$-modules. Note that the space of $C$-invariants on $Y$ and $Z$ are each 2-dimensional and the space of $N$-invariants on $Y$ and $Z$ are each 1-dimensional. The invariants of $C$ on $Z$ are contained in the $\nu(V)$-eigenspace of $\tau$. Since $\tau$ interchanges $V \otimes x V$ and $x V \otimes V$, we see that $\tau$ has two eigenvalues on the invariants of $C$ on $Y$. Thus, $\tau$ has a 3-dimensional $\nu(V)$-eigenspace and 1-dimensional $\nu(V)$-eigenspace on the $C$-invariants on $X$.

First suppose that each $U_{i}$ is self dual as a $k N$-module. Then $\nu\left(U_{i}\right)=\nu(V)$ for each $i$ and so $\nu(W)=2 \nu(V)$, and we need to show that $\gamma=\nu(V)$ in this case. We can also view $X=\oplus U_{i} \otimes U_{j}$; then we see that the $N$-invariants are contained in $U_{1} \otimes U_{1} \oplus U_{2} \otimes U_{2} ;$ and that $\tau$ acts on this space as $\nu(V)=\nu(W)-\nu(V)$.

Finally, suppose that each $U_{i}$ is not self dual. Thus, $\nu(W)=0$. We need to show that $\gamma=-\nu(V)$ in this case. On $Z, \tau$ acts via $\nu(V)$ on the $C$-fixed points and so on the $N$-fixed points. Since $W$ is self dual (as $V$ is), this implies that $U_{2}=U_{1}^{*}$. Thus, we see that $\tau$ has both eigenvalues on the 2-dimensional space of $N$-fixed points on $X$, whence $\tau(f)=-\nu(V) f$.

It is straightforward to see that this implies the following theorem, which is a refinement of [14, Corollary 6.2] if $k=\mathbb{C}$ but is new if $k$ has positive characteristic $p$ dividing $|G|$. 
Proposition 2.10. Let $G$ be a finite group. Then $\nu(\hat{V})=1$ for all irreducible $D(G)$-modules $\hat{V}$ if and only if:

(1) When $g^{2}=1, \nu(V)=1$ for all irreducible $C_{g}$-modules $V$.

(2) When $g^{2} \neq 1,\left|N_{g}: C_{g}\right|=2$ and for any irreducible $C_{g}$-module $V$,

(a) $V^{x} \cong V^{*}$ for all $x \in N_{g} \backslash C_{g}$, where $V^{x}$ is the conjugate module to $V$,

(b) if $V$ is not self dual, then $V_{C_{g}}^{N_{g}}$ has Schur indicator +1 ,

(c) if $\nu(V)=+1$, then each constituent of $V_{C_{g}}^{N_{g}}$ has Schur indicator +1 ,

(d) if $\nu(V)=-1$, then neither constituent of $V_{C_{g}}^{N_{g}}$ is self dual.

Proof. If $g^{2}=1$, this is clear. This also implies that every element of $\langle g\rangle$ is conjugate to its inverse.

So consider the case where $g^{2} \neq 1$ and fix $x \in N_{g}$ with $x g x^{-1}=g^{-1}$. Recall that $V^{x}=V$ as a $k$-space, but with new action $g \cdot{ }_{x} v=x g x^{-1} \cdot v$, for all $g \in C_{g}$, $v \in V^{x}$.

We have already seen that $V^{x} \cong V^{*}$ if and only if all indicators for $D(G)$ are non-zero. Note that the condition that $V^{x} \cong V^{*}$ implies that $V_{C_{g}}^{N_{g}}$ is self dual. So we assume that this is the case.

Suppose that $V$ is not self dual. Then $\nu(\hat{V})=\nu\left(V_{C_{g}}^{N_{g}}\right)$. So we may assume that $V$ is self dual and $V \cong V^{x}$.

Now it follows by Frobenius reciprocity that the induced module is a direct sum of two irreducible modules. Note that the sum of the characters of the two irreducible modules vanishes outside $C_{g}$ (because the sum of the modules is an induced module). In particular, one is self dual if and only if the other one is self dual. So if neither is self dual, then $\nu(\hat{V})=-\nu(V)$, and the theorem holds.

If they are both self dual, then the Schur indicators for each of the two modules must be equal to $\nu(V)$, and so $\nu(\hat{V})=\nu(V)$. This completes the proof.

In particular, this shows:

Corollary 2.11. Let $G$ be a finite group and $k$ an algebraically closed field of characteristic not 2. Assume that $g$ is conjugate to $g^{-1}$ for all $g \in G$. If $x \in$ $N_{g} \backslash C_{g}$ implies that $V^{*} \cong V^{x}$ for all irreducible $k C_{g}$-modules and $\nu(W)=1$ for all irreducible $k N_{g}$-modules, then $D(G)$ is totally orthogonal.

The previous results also imply:

Corollary 2.12. Let $G$ be a finite group. Then $\nu(\hat{V})= \pm 1$ for all irreducible $D(G)$-modules $\hat{V}$ if and only if:

(1) when $g^{2}=1$, every irreducible module of $C_{g}$ is self dual;

(2) when $g^{2} \neq 1,\left|N_{g}: C_{g}\right|=2$, and for any irreducible $C_{g}$-module $V$, we have $V^{x} \cong V^{*}$ for all (any) $x \in N_{g} \backslash C_{g}$.

Remark 2.13. G. Mason has also observed that Corollary 2.12 for $k=\mathbb{C}$ can be obtained using the methods of [14] (private communication).

Remark 2.14. The condition that $V^{x} \cong V^{*}$ for all $\mathbb{C}\left[C_{g}\right]$ modules $V$ with $x \in N_{g} \backslash C_{g}$ is equivalent to saying that $x$ inverts all conjugacy classes of $C_{g}$ (i.e. if $y \in C_{g}$, then $y^{x}$ is conjugate to $y^{-1}$ in $C_{g}$ ). 


\section{SCHUR INDICES I}

We recall some basic facts about Schur indices. See [5] for details. Let $G$ be a finite group and $k$ a field of characteristic 0 . Let $V$ be an absolutely irreducible $G$-module over the algebraic closure of $k$ with character $\chi$. Let $k^{\prime}$ be the extension field of $k$ generated by the values of $\chi$. The Schur index $m_{\chi}$ is the smallest positive integer such that there is a $k^{\prime} G$-module $W$ with character $m_{\chi} \chi$.

The basic fact that we will require about this is (cf. [5, Lemma 9.1]):

Lemma 3.1. Suppose $W$ is a $k G$-module with character $\theta$. If $\chi$ is an irreducible character of $G$ with values in $k$, then $m_{\chi}$ divides $(\theta, \chi)$.

This has the following easy consequence:

Lemma 3.2. Let $N$ be a subgroup of index 2 in the finite group $G$. Let $k$ be a subfield of $\mathbb{C}$. Let $V$ be an irreducible $\mathbb{C} N$-module with character $\chi$ having values in k. Set $W=V_{N}^{G}$.

(1) If each irreducible constituent of $W$ is defined over $k$, then $V$ is defined over $k$.

(2) If $V$ is defined over $k$ and each irreducible constituent of $W$ has character values in $k$, then they are defined over $k$.

Proof. The key point is that $W$ is multiplicity free. Indeed, by Frobenius reciprocity, either $W$ is irreducible and $W_{N}$ is a direct sum of two non-isomorphic modules ( $V$ and $V^{x}$ for $\left.x \in G \backslash N\right)$ or $W$ is the direct sum of two non-isomorphic $G$-modules which are isomorphic as $N$-modules.

(1) If $W$ is not irreducible and is defined over $k$, then each constituent is isomorphic to $V$ as an $N$-module, whence $V$ is defined over $k$.

If $W$ is irreducible, then by Frobenius reciprocity, $1=\left(\chi_{N}^{G}, \chi_{N}^{G}\right)_{G}=\left(\chi, \chi_{N}^{G}\right)$. Thus, the previous result implies that $m_{\chi}=1$, as required.

(2) If $W$ is irreducible, then since it is induced from a module defined over $k$, it is defined over $k$. If $W$ is not irreducible, then it is the sum of two non-isomorphic irreducible modules each with character in $k$. By the previous result, the Schur index of each constituent is 1 , whence they are each defined over $k$.

An immediate consequence is the following:

Corollary 3.3. Let $N$ be a subgroup of index 2 in the finite group $G$. Let $k$ be a field of characteristic 0. Suppose that all characters of $N$ and $G$ are defined over $k$. Then $k$ is a splitting field for $N$ if and only if it is a splitting field for $G$.

Let $C$ be a subgroup of index 2 in the finite group $N$. Pick $x \in N \backslash C$ and assume that $x$ inverts all conjugacy classes of $C$. We consider two conditions:

DC1 If $V$ is an irreducible $C$-module with $\nu(V)=0$, then $V_{C}^{N}$ is irreducible with $\nu\left(V_{C}^{N}\right)=+1$. Moreover, if $\nu(V)= \pm 1$, then $V$ has two (distinct) extensions to $N$, both with Schur indicator +1 if $\nu(V)=+1$ and 0 if $\nu(V)=-1$ (this can be summarized by saying that $\nu\left(V_{C}^{N}\right)-\nu\left(V_{C}\right)=+1$ for all irreducible $C$-modules).

DC2 $\nu(W)=+1$ for all irreducible $N$-modules.

It is an easy exercise to show that DC2 implies DC1. We show that these properties behave nicely with respect to direct product. 
Lemma 3.4. Let $N_{i}, 1 \leq i \leq t$, be finite groups with subgroups $C_{i}$ of index 2 . Fix $x_{i} \in N_{i} \backslash C_{i}$. Assume that $x_{i}$ inverts all conjugacy classes of $C_{i}$. Let $N=$ $N_{1} \times \ldots \times N_{t}$ and $C=C_{1} \times \ldots \times C_{t}$. Set $H=\langle C, x\rangle$ where $x=x_{1} \cdots x_{t}$.

(1) $x$ inverts all conjugacy classes in $C$.

(2) If $C_{i}<N_{i}$ satisfies DC1 or DC2 for each $i$, then so does $C<H$.

Proof. The first statement is clear. This shows that if $V$ is an irreducible $C$-module and is not self dual, then $V^{x} \cong V^{*}$ is not isomorphic to $V$.

We now prove (2). By induction, we may reduce to the case that $t=2$. If $\nu(W)=+1$ for all irreducible $N_{i}$-modules (i.e. DC2 holds), then by Corollary 3.3 , it suffices to show that all characters of $H$ are real valued. Clearly, every element of $C$ is conjugate to its inverse in $H$. Suppose that $y:=\left(y_{1}, y_{2}\right) \in H$ and $y_{i}$ is not in $C_{i}$. Then $y_{i}$ is conjugate to its inverse via an element of $C_{i}$, since all representations of $N_{i}$ are self dual. Then $y^{g}=y^{-1}$ for some $g \in C$, whence $y$ is conjugate to its inverse in $H$.

We now show that DC1 extends as well.

Suppose that $V$ is an irreducible $C$-module. Write $V=V_{1} \otimes V_{2}$. So $\nu(V)=$ $\nu\left(V_{1}\right) \nu\left(V_{2}\right)$.

If $\nu\left(V_{1}\right)=\nu\left(V_{2}\right)=1$, then both $V_{i}$ extend to irreducible $N_{i}$-modules all with Schur indicator +1 , and so therefore the same is true for each constituent of $V_{C}^{H}$.

If $\nu\left(V_{1}\right)=\nu\left(V_{2}\right)=-1$, then $\nu(V)=+1$, and so $V$ is defined over $\mathbb{R}$. By hypothesis, $U_{i}:=V_{i_{i}}^{N_{i}}$ is a direct sum of two irreducible modules which are not self dual for $N_{i}$. Let $X_{i}$ and $Y_{i}$ be the two irreducible constituents. Since $U_{i}$ is self dual, $Y_{i}=X_{i}^{*}$, and since the character of $U_{i}$ vanishes outside $C_{i}$, it follows that the character of $X_{i}$ (and of $Y_{i}$ ) is purely imaginary on $N_{i} \backslash C_{i}$. Thus, the character of $X_{i}$ on $H$ is real valued. By Lemma 3.2, this implies that both $X_{i}$ and $Y_{i}$ are defined over $\mathbb{R}$.

If $\nu\left(V_{1}\right)=-\nu\left(V_{2}\right) \neq 0$, then $\nu(V)=-1$, and we need to show that an extension of $V$ to $H$ is not self dual. Assume that $\nu\left(V_{1}\right)=-1$. Then we can choose $c_{i} \in C_{i}$ such that the character of $x_{i} c_{i}$ is non-zero and purely imaginary for $i=1$ and real for $i=2$ (the character cannot vanish outside $C_{i}$ by Frobenius reciprocity). Then the character value of $\left(c_{1} x_{1}, c_{2} x_{2}\right)$ on any extension of $V$ is purely imaginary and non-zero (being the product of the character values for the extensions of $V_{1}$ and $V_{2}$ ), as required.

So we may assume that $\nu(V)=\nu\left(V_{1}\right)=0$. Then $W:=V_{C}^{H}$ is irreducible and self dual. By hypothesis, $W_{H}^{N}=V_{C}^{N}$. So $U_{1}$ is irreducible as an $N_{1}$-module and $\nu\left(U_{i}\right)=1$. Note that $C_{1}$ has a 1-dimensional fixed space on both $\operatorname{Sym}^{2}\left(U_{1}\right)$ and $\bigwedge^{2}\left(U_{1}\right)$ and that $H$ is trivial on $\operatorname{Sym}^{2}\left(U_{1}\right)$ and $x_{1}$ acts as -1 on $\bigwedge^{2}\left(U_{1}\right)$.

Note that $W_{H}^{N}=V_{C}^{N}=U_{1} \otimes U_{2}$. If $\nu\left(V_{2}\right) \geq 0$, then each constituent of $U_{2}$ has Schur indicator +1 , and so $W_{H}^{N}$ is defined over $\mathbb{R}$, whence by Lemma 3.2, $W$ is.

Suppose that $\nu\left(V_{2}\right)=-1$. Then $C_{2}$ has a fixed point on $\bigwedge^{2}\left(V_{2}\right)$. Now $V_{2}$ can be viewed as a non-self dual module for $N_{2}$ (in two distinct ways) and $W=$ $U_{1} \otimes V_{2}$. Thus, $x_{2}$ acts on $\bigwedge^{2}\left(V_{2}\right)$ by -1 . So we see that $x$ has a fixed point on $\bigwedge^{2}\left(U_{1}\right) \otimes \bigwedge^{2}\left(V_{2}\right) \leq \operatorname{Sym}^{2}\left(U_{1} \otimes V_{2}\right)$. Thus, $\nu(W)=+1$, as required.

\section{Some Reductions}

Let $G$ be a finite group, and consider the following three properties of $G$ :

(R1) Each irreducible $D(G)$-module has Schur indicator \pm 1 ; 
(R2) Each irreducible $D(G)$-module has Schur indicator +1 ;

(R3) Each irreducible $D(G)$-module has Schur indicator +1 , and for each $g \in G$, every $N_{g}$-module has Schur indicator +1 .

It is clear that (R3) implies (R2) implies (R1). None of the implications can be reversed. For example, $G=Q_{8}$ satisfies (R1) but not (R2). The Weyl groups of types $F_{4}, E_{6}, E_{7}, E_{8}$ and the real reflection group $H_{4}$ satisfy (R2) but not (R3); this can be seen using Magma. We will see that the classical Weyl groups do satisfy (R3).

We describe the groups $G$ for which $D(G)$ satisfies (R3) using Proposition 2.10,

Lemma 4.1. Let $G$ be a finite group. Then $D(G)$ (over $\mathbb{C}$ ) satisfies (R3) if and only if for each $g \in G, \nu(V)=+1$ for all irreducible $N_{g}$-modules $V$, and if $x \in G$ inverts $g$, then $x$ inverts all $C_{g}$ conjugacy classes.

We first note:

Lemma 4.2. Suppose that $G=N \rtimes H$, a semidirect product of a normal subgroup $N$ and a complement $H$. If $G$ satisfies (Ri) with $1 \leq i \leq 3$, then so does $H$.

Proof. Let $h \in H$. Then $C_{H}(h)$ is a homomorphic image of $C_{h}$ and similarly for $N_{h}$. Thus, the modules considered in computing the Schur indicators have already arisen in the computation for $G$.

Proposition 4.3. Let $G$ be a finite group. Assume that $G$ is a direct product of groups $G_{j}$. Then for each $i G$ satisfies (Ri) if and only if each $G_{j}$ does.

Proof. It suffices to consider a direct product of 2 groups. The previous lemma implies the forward implication. So assume that each direct factor satisfies (R1).

So assume that $G=A \times B$ and $g=(a, b)$. Then $C_{g}=C_{a} \times C_{b}$, and we can take $t=(u, v)$ inverting $g$. Then $t$ clearly inverts every class of $C_{g}$. This shows that (R1) holds, using Corollary 2.12, So we are in the case of (R2) or (R3). Moreover, every $D(G)$-module has a non-zero Schur indicator (since the result holds for (R1)).

If $g^{2}=1$, then since $C_{g}$ is a direct product, the result is clear.

So we may assume that $a^{2} \neq 1$. Let $V$ be an irreducible $C_{g}$ module. So $V=$ $V_{A} \otimes V_{B}$, where $V_{A}$ and $V_{B}$ are irreducible $C_{a}$ and $C_{b}$-modules, respectively. If $b^{2}=1$, then $N_{g}=N_{a} \times C_{b}=N_{a} \times N_{b}$ and all irreducible modules are tensor products. Since $B$ satisfies (R2), all Schur indices of $C_{b}$-modules are +1 . Since $A$ satisfies (R2) or (R3), it is trivial to see that all Schur indices for the $H$-modules occurring are +1 and that moreover if (R3) holds for $A$ and $B$, then every $N_{g^{-}}$ module is real.

So we may assume that $a^{2} \neq 1 \neq b^{2}$. Let $N=N_{a} \times N_{b}$. Now the result follows by Lemma 3.4 .

We end this section by showing that being totally orthogonal in characteristic zero implies the same result for odd characteristic. Let $k$ be an algebraically closed field of odd characteristic. A result of Thompson [29] asserts that if $V$ is an irreducible $k G$-module of a non-zero Schur indicator, then $V$ is a composition factor (of odd multiplicity) in the reduction mod $p$ of an irreducible $\mathbb{C} G$-module with the same Schur indicator as $V$.

Theorem 4.4. Let $G$ be a finite group. Let $k$ be an algebraically closed field of odd characteristic. 
(1) If all irreducible $\mathbb{C} G$-modules have Schur indicator +1 , the same is true for all irreducible $k G$-modules.

(2) If all irreducible $\mathbb{C} G$-modules have Schur indicator at least 0 , the same is true for all irreducible $k G$-modules.

(3) If all irreducible $\mathbb{C} G$-modules are self dual, the same is true for all irreducible $k G$-modules.

Proof. (3) is clear since the hypothesis implies all elements of $g$ are conjugate to their inverse.

Now consider (2). By the result of Thompson mentioned above, there are no irreducible $k G$-modules $V$ with $\nu(V)=-1$. So (2) follows immediately.

Now (1) follows by (2) and (3).

We use the previous result to show that $D(G)$ being totally orthogonal in characteristic zero implies the same result for odd positive characteristic.

Corollary 4.5. Let $G$ be a finite group and $k$ an algebraically closed field of odd characteristic $p$. Let $D(G)$ denote the Drinfel'd double of $G$ over $\mathbb{C}$ and $D_{k}(G)$ the Drinfel'd double of $G$ over $k$.

(1) If $D(G)$ is totally orthogonal, then so is $D_{k}(G)$.

(2) If $D(G)$ satisfies (R3), then does does $D_{k}(G)$.

(3) If $D(G)$ satisfies (R1), then does does $D_{k}(G)$.

Proof. Assume that $D(G)$ is totally orthogonal. Note that this implies $G$ is totally orthogonal. In particular, every element is conjugate to its inverse. Fix $g \in G$.

If $g^{2}=1$, then $C_{g}$ is totally orthogonal in characteristic 0 and so by the previous result also over $k$.

Suppose that $g^{2} \neq 1$. Then, as noted above, $\left[N_{g}: C_{g}\right]=2$ and $x \in N_{g} \backslash C_{g}$ implies that $x$ inverts all conjugacy classes of $C_{g}$ (by the assumption for characteristic 0) and so $V^{*} \cong V^{x}$ for all irreducible $k C_{g}$-modules $V$.

Let $V$ be an irreducible $k C_{g}$-module.

If $V$ is not self dual, then $W:=V_{C_{g}}^{N_{g}}$ is self dual for $N_{g}$ and $\nu(\hat{V})=\nu(W)$. We claim that $\nu(W)=1$.

By Thompson's result, $W$ is contained in the reduction of an irreducible $\mathbb{C} N_{g^{-}}$ module $U$ with $\nu(U)=\nu(W)$ and the multiplicity of $W$ in the reduction of $U$ is odd. Suppose that $U$ is irreducible as a $C_{g}$-module. Since $D(G)$ is totally orthogonal, it follows that $1=\nu\left(U_{C_{g}}^{N_{g}}\right)-\nu\left(U_{C_{g}}\right)=\nu\left(U_{C_{g}}\right)=\nu\left(U_{N_{g}}\right)=1$. Since $\nu\left(U_{N_{g}}\right)=\nu(W)$, the claim holds in this case.

If $U$ is not irreducible as a $C_{g^{-}}$-module, then $U=Y_{C_{g}}^{N_{g}}$ for some irreducible $C_{g^{-}}$ module $Y$. Then, $V$ is a constituent of the reduction of $Y$ of odd multiplicity. So if $Y$ is self dual, it follow that $V$ is, a contradiction. Thus, $V$ is not self dual and so $D(G)$ totally orthogonal implies that $\nu(W)=1$.

Suppose that $V$ is self dual and so $\nu(V) \neq 0$. Let $W$ be an irreducible $\mathbb{C} C_{g^{-}}$ module with $\nu(W)=\nu(V)$ such that $V$ occurs with odd multiplicity in the reduction of $W$. Then $W$ can be viewed as an $N$-module. Since $D(G)$ is totally orthogonal, either $\nu(W)=1$ both as an $N_{g^{-}}$and $C_{g^{-}}$-module or $\nu(W)=-1$ as a $C_{g^{-}}$-module and $\nu(W)=0$ as an $N_{g}$-module.

First suppose that $\nu(W)=+1$ (and so also for $N_{g}$ ). The reduction of $W$ has one of the extensions of $V$ occurring with odd multiplicity (since this is true of $V$ in the reduction of $W$ as a $C_{g}$-module). Since $W$ is self dual, the composition factors 
in the reduction must be closed under duals, whence the $N_{g}$-module $V$ must be self dual and therefore must satisfy $\nu(V)=+1$.

Suppose that $\nu(V)=-1$. Since $\nu(U) \neq-1$ for any irreducible $\mathbb{C} N_{g}$-module, by Thompson's result, $\nu(V) \neq-1$ as a $k N_{g}$-module, whence $\nu(V)=0$.

We have now shown that the necessary (and sufficient) conditions for $D(G)$ to be totally orthogonal over $k$ are satisfied.

If in addition, $D(G)$ satisfies (R3), we only need to verify that $k N_{g}$ is totally orthogonal, but this is Theorem 4.4 .

The proof for (R1) is analogous, and we leave this as an exercise to the reader (this is not used in the sequel).

\section{Abelian subgroups of ReAl REFlection groups}

We now prove Theorem 1.2 for the classical Weyl groups. This also proves that every irreducible representation of $C_{g}$ is self dual, but we will prove more later. In fact, we prove a stronger result. We first consider the case of symmetric and alternating groups.

Lemma 5.1. Let $W=\operatorname{Sym}_{n}$ and let $J$ be an abelian subgroup of $W$. Let $r$ be an integer prime to $|J|$. Then there exists $x \in W$ such that $x^{-1} y x=y^{r}$ for all $y \in J$. Moreover,

(1) If $r=-1$, then we can choose $x$ to be an involution.

(2) If all non-trivial orbits of $J$ have even size, then we can choose $x \in \mathrm{Alt}_{n}$.

(3) If $r=-1$ and all non-trivial orbits of $J$ have even size, we can choose $x$ to be an involution in $\mathrm{Alt}_{n}$.

Proof. We prove the first statement. By induction, it suffices to assume that $J$ is transitive and so acts regularly and has order $n$. Let $X=\operatorname{Aut}(\mathrm{J})$. Note that $H:=J . X$ embeds in $\operatorname{Sym}_{n}$ by letting $H$ act on the cosets of $X$. Then $J$ maps onto a transitive abelian subgroup of $\mathrm{Sym}_{n}$, and the result follows.

If $r=-1$, the same proof shows that $x$ can be chosen to be an involution.

Suppose that all non-trivial orbits of $J$ have even order. By induction, we may reduce to the case that $J$ is transitive of even order. If $J$ is not contained in $\mathrm{Alt}_{n}$, then we may replace $x$ by $x s$ for some $s \in J$ to allow us to choose $x$ in either coset. So $J \leq \mathrm{Alt}_{n}$ which implies that the Sylow 2-subgroup of $J$ is not cyclic.

So we can write $J=C \times D \leq S_{c} \times S_{d}$ with $C$ and $D$ of even order and $n=c d$. Now choose $x_{C}$ and $x_{D}$ acting on $C$ and $D$ respectively in the appropriate manner and take $x=x_{C} x_{D}$. We may assume that $c$ is even, and so by induction $x_{C} \in A_{c}$, and so $x_{C}$ is also in $A_{n}$. Since $c$ is even, $x_{D}$ is also in $A_{n}$ (whether or not $x_{D} \in A_{d}$ ). Thus, $x \in \mathrm{Alt}_{n}$ as required. Moreover, if $r=-1$, by construction $x^{2}=1$.

We can now handle the classical Weyl groups.

Theorem 5.2. Let $W$ be a Weyl group, $J$ be an abelian subgroup and $r$ be an integer prime to the order of $J$. Then there exists $x \in W$ such that $x^{-1} y x=y^{r}$ for all $y \in J$. If $r=-1$, then we can choose $x$ to be an involution.

Proof. We first note that we may and do assume that $r$ is odd. For if $J$ has even order, then certainly $r$ is odd. If $J$ has odd order, we may replace $r$ by $r+|J|$. The case $W=\operatorname{Sym}_{n}$ follows by the previous result.

Next we consider $W$ of type $B_{n}$. Let $W_{0} \cong D_{n}$ be a subgroup of index 2 in $W$. We show that we can take $x \in W_{0}$ with the appropriate properties. 
We can view $W$ as the centralizer of a fixed point free involution $z \in \operatorname{Sym}_{2 n}$. Note that $W_{0}=W \cap \operatorname{Alt}_{2 n}$.

Let $J$ be an abelian subgroup of $W$. There is no harm in assuming that $z \in J$ (and so all orbits of $J$ have even order). The result now follows by the previous result.

By the result for the symmetric group, we can find $x \in$ Alt $_{2 n}$ with $x^{-1} y x=y^{r}$ for all $y \in J$. In particular, $x$ commutes with $z$ and so $x \in W \cap \operatorname{Alt}_{2 n}=W_{0}$. Moreover, if $r=-1$, we can take $x$ to be an involution in $W_{0}$. This completes the proof for the classical Weyl groups.

If $W$ is exceptional, then Breuer [3] has used GAP to check that the result holds.

Corollary 5.3. Let $E$ be an elementary abelian 2-subgroup of the Weyl group $W$. Then all characters of $C_{W}(E)$ are rational valued.

Proof. Let $g \in C_{W}(E)$ and let $r$ be an odd integer prime to the order of $g$. Then $J:=\langle E, g\rangle$ is abelian, and so there exists $x \in W$ with $x_{a x}{ }^{-1}=a^{r}$ for all $a \in J$. Thus, $x \in C_{W}(E)$, and so $g$ is conjugate to any power of $g$ prime to its order, whence all characters of $C_{W}(E)$ are rational valued on $g$.

For general real reflection groups, we observe:

Theorem 5.4. Let $W$ be a finite real reflection group and let $J$ be an abelian subgroup of $W$. There exists an involution in $W$ that inverts $J$.

Proof. Clearly, we may assume that $W$ is irreducible. If $W$ is a Weyl group, the result follows by the previous theorem. If $W$ is dihedral or $W \cong H_{3} \cong A_{5} \times \mathbb{Z} / 2$, the result is clear.

The remaining groups are $H_{4}, F_{4}, E_{6}, E_{7}$ and $E_{8}$. In these cases, we use computer calculations to verify the result (see $[\underline{3}$ for details).

Corollary 5.5. Let $W$ be a real reflection group.

(1) If $g^{2}=1$, then every element of $C_{g}$ is a product of two involutions, and, in particular, all irreducible representations of $C_{g}$ are self dual.

(2) If $g^{2} \neq 1$ and $x \in N_{g} \backslash C_{g}$, then $x$ inverts all conjugacy classes of $C_{g}$.

Proof. Suppose that $g^{2}=1$ and $y \in C_{W}(g)$. By the previous result, there exists an involution $x \in C_{W}(g)$ inverting $y$. Then $y=x(x y)$ is a product of two involutions in $C_{W}(g)$. This implies that all irreducible representations of $C_{W}(g)$ are self dual and proves (1).

Fix $x \in N_{g}$. Let $y \in C_{g}$. By the previous result, there exists $x^{\prime} \in N_{g}$ inverting $y$. Thus, $x^{\prime}=x c$ with $c \in C_{g}$. Thus, $y^{-1}=y^{x c}$, and so the $C_{g}$ conjugacy class of $y^{x}$ is the $C_{g}$ conjugacy class of $y^{-1}$, as asserted.

Using Corollary 2.12, this gives a fairly quick proof that the indicators for the simple modules of the Drin'feld double of a real reflection group are at least nonzero.

We have seen that all characters of $C_{W}(E)$ are rational valued for $E$ an elementary abelian 2-group of the Weyl group $W$. In fact, it is true that all representations of $C_{W}(E)$ are defined over $\mathbb{Q}$. We give the proof for the classical Weyl groups below. See [7] for the case of the exceptional Weyl groups. 
Theorem 5.6. Let $W$ be either a Weyl group or an alternating group. Let $E$ be an elementary abelian 2-subgroup, and if $W=$ Alt $_{n}$ assume that $E$ has no fixed points. Then all representations of $C_{W}(E)$ are defined over $\mathbb{Q}$.

Proof. First suppose that $W=\operatorname{Sym}_{n}$. If $E=1$, the result is clear. If $1 \neq x \in E$ has fixed points, then $C_{W}(E) \leq C_{W}(x)$ is contained in a direct product of two smaller symmetric groups, so the centralizer will be the direct product of centralizers in these smaller symmetric groups and the result follows by induction. So we may assume that all orbits of $E$ are regular. So $n=m t$, where $m=|E|$ and $t$ is the number of orbits of $E$. Then $C_{W}(E) \cong E \imath S_{t}$. It is straightforward to see that all representations of this group are defined over $\mathbb{Q}$ (see the proof of Lemma 6.1 for a more general result).

Now suppose that $E$ has no fixed points and $W=\operatorname{Alt}_{n}$. Let $G=\operatorname{Sym}_{n}$. If $C_{W}(E)=C_{G}(E)$, the result follows. Otherwise, $\left[C_{G}(E): C_{W}(E)\right]=2$. By Lemma 5.1, all characters of $C_{W}(E)$ are rational, and so by Lemma 3.2 and the result for $C_{G}(E)$, all representations of $C_{W}(E)$ are defined over $\mathbb{Q}$.

Next suppose that $W$ is a Weyl group of type $B_{n}$. Then $W$ is the centralizer of a fixed point free involution $z \in G:=\operatorname{Sym}_{2 n}$. We may as well assume that $z \in E$. Then $C_{G}(E)=C_{W}(E)$, and so the result holds.

If $W$ has type $D_{n}$ and $n$ is odd, then $W \times Z \cong B_{n}$, where $Z$ is the center of $B_{n}$ of order 2 , and so the result follows by the result for type $B$. If $W$ has type $D_{n}$ and $n$ is even, then $W$ contains the central involution $z$ of $B_{n}$ and again we may assume that $z \in E$. Let $H=\operatorname{Alt}_{2 n}$. So $W=B_{n} \cap H$ and $C_{W}(E)=C_{H}(E)$, and so the result follows by the result for alternating groups.

If $W$ is exceptional, this is proved in 7 .

For the other real reflection groups, we know the following:

Theorem 5.7. Let $W$ be a finite real reflection group and $E$ an elementary abelian 2-subgroup of $W$. All representations of $C_{W}(x)$ are defined over $\mathbb{R}$.

Proof. Clearly, we may assume that $W$ is irreducible.

If $W$ is a Weyl group, this follows by the previous result.

If $W$ is an irreducible real reflection group other than a Weyl group, this follows by inspection (these are just dihedral groups, $H_{3}$ and $H_{4}$ ), whence (1) follows.

\section{SOME REAL GROUPS}

Recall that a Young subgroup of $S_{m}$ is the largest subgroup of $S_{m}$ with a given set of orbits (so of the form of a direct product of symmetric groups of size $m_{i}$ with $\left.\sum m_{i}=m\right)$.

Lemma 6.1. Let $A$ be a finite abelian group of exponent $n$ and $m$ a positive integer. Set $B=A^{(m)}$. Let $S_{m}$ act on $B$ in the obvious way (permuting the $m$ copies of $A)$. Let $\tau$ be the involution acting as inversion on $B$. Note that $\tau$ commutes with the action of $S_{m}$ on $B$. Set $G=B \rtimes\left(S_{m} \times\langle\tau\rangle\right)$ and $G_{0}=B \rtimes S_{m}$. Let $L$ be the real subfield of $K:=\mathbb{Q}[\zeta]$, where $\zeta$ is a primitive $n$th root of unity. Then every representation of $G$ is defined over $K$ and every representation of $G_{0}$ is defined over $L$.

Proof. We leave the second statement as an exercise (it is essentially the first case of the proof of the first statement given below). 
Let $V$ be an irreducible $G$-module. Let $V_{\gamma}$ be the $\gamma$ eigenspace for $B$ for some $\gamma \in B^{*}$, the dual group of $B$, and assume that this is non-zero. Then $G$ permutes these eigenspaces and the stabilizer $S$ of this eigenspace satisfies one of the following possibilities:

(1) $S=B Y$, where $Y$ is a Young subgroup;

(2) $\gamma^{2}=1$ (or equivalently, $\gamma=\gamma^{*}$ ) and $S=B(Y \times\langle\tau\rangle)$; or

(3) $S=B(\langle Y, g \tau\rangle)$, where $g \in S_{m}$ is an involution and $\gamma^{g}=\gamma^{*}$.

The first case is where $\gamma$ and $\gamma^{*}$ are not in the same orbit under $S_{m}$, the third case is where $\gamma \neq \gamma^{*}$ but $\gamma$ and $\gamma^{*}$ are distinct but in the same orbit (and we can always choose an involution which takes $\gamma$ to $\gamma^{*}$ ) and the second case is where $\gamma$ is self dual.

Consider the first case. Let $U=V_{\gamma} \oplus V_{\gamma^{*}}=V_{\gamma} \oplus \tau V_{\gamma}$. Then the stabilizer of $U$ is $T:=B(Y \times\langle\tau\rangle)$. By construction, the image $D$ of $B\langle\tau\rangle$ acting on $U$ is a dihedral group. Thus, the image of $T$ factors through $D \times Y \times \mathbb{Z} / 2$. Since every representation of this group is defined over $L$, it follows that $U$ is defined over $L$. Since $V$ is induced from $U$, the same is true for $V$.

The second case is similar. Let $U=V_{\gamma}$. Thus $S$ acts on $V_{\gamma}$ as a homomorphic image of $Y \times \mathbb{Z} / 2 \times \mathbb{Z} / 2$, and the representation is defined over $\mathbb{Q}$.

Next consider the third case. Write $\gamma=\left(\gamma_{1}, \ldots, \gamma_{m}\right)$. The hypothesis implies that for each $\alpha$ in the dual group $A^{*}$ of $A$, the number of $i$ with $\gamma_{i}=\alpha$ is the same as the number of $i$ with $\gamma_{i}=\alpha^{*}$. It follows that $S$ has a normal subgroup that is the direct product of symmetric groups and that $g$ and $\tau$ are commuting involutions with $g \tau$ an involution which either commutes with a direct factor or interchanges two direct factors (depending upon whether the corresponding character is self dual or not).

Let $U=V_{\gamma} \oplus V_{\gamma^{*}}$. The stabilizer of $U$ is $T:=\langle S, \tau\rangle$. Note that $g \tau$ commutes with the image of $B$ on $U$ and so $T$ acts on $U$ as a homomorphic image of $D \times\langle Y, g \tau\rangle$. Thus, it suffices to prove that $\langle Y, g \tau\rangle$ has all representations defined over $F$. Note that $Y$ is a direct product of symmetric groups and $g \tau$ either commutes with a direct factor or interchanges two factors. So $\langle Y, g \tau\rangle$ contains a direct product $Y_{1} \times \ldots \times Y_{t}$, where each $Y_{i}$ is either a symmetric group or a direct product of 2 symmetric groups and is contained in a direct product of $Z_{i}, 1 \leq i \leq t$ where $Z_{i} \cong Y_{i} \times \mathbb{Z} / 2$ or is a wreath product $U\ulcorner\mathbb{Z} / 2$ where $U$ is a symmetric group. Since all representations of $Y_{i}$ and $Z_{i}$ are defined over $\mathbb{Q}$, the same is true for $\langle Y, g \tau\rangle$ by Lemma 3.2. This completes the proof.

We will need the following result to deduce the results for Weyl groups of type D from those of type B.

Lemma 6.2. Let $A$ be a finite cyclic group of even order $r$ and $m$ a positive integer. Set $B=A^{(m)}$. Then $S_{m}$ acts on $B$ in the obvious way (permuting the $m$ copies of $A$ ). Let $A^{2}$ denote the group of squares in $A$. Let $\tau$ be the involution acting as inversion on $B$. Set $G=B \rtimes\left(S_{m} \times\langle\tau\rangle\right)$. Let $B_{0}$ be the subgroup of $B$ of index 2 that is normal in $G$ (i.e. $\left.B_{0}=\left\{\left(a_{1}, \ldots, a_{m}\right) \in B \mid a_{1} \cdots a_{m} \in A^{2}\right\}\right)$. Let $G_{0}=B_{0}\left(S_{m} \times\langle\tau\rangle\right)$. Let $L$ be the real subfield of $K:=\mathbb{Q}\left[\zeta_{r}\right]$. Then

(1) every representation of $B_{0} \rtimes S_{m}$ is defined over $K$;

(2) every representation of $G_{0}$ is defined over $L$; and

(3) $\tau$ inverts all conjugacy classes in $B_{0} S_{m}$. 
Proof. View $A$ as subgroup of $\operatorname{Sym}_{r m}$ acting semiregularly. Then $B_{0} S_{m}$ is isomorphic to the centralizer of $A$ in Alt $_{r m}$ and $G$ is the subgroup of Alt ${ }_{r m}$ consisting of all elements that either centralize $A$ or act as an inversion on $A$. We have already proved (3) in this context.

It is an easy exercise to show that if $r$ is 1 modulo $m$ and relatively prime to the order of an element $g \in B S_{m}$, then $g^{r}$ and $g$ are conjugate via $B_{0} S_{m}$ (indeed, this follows by our result for abelian subgroups of the Weyl group of type D).

Using Lemma 3.2 and the previous result implies that $B_{0} S_{m}$ has all representations defined over $K$.

In order to prove the second statement, by Lemma 3.2 and the previous result it suffices to prove that every representation of $G_{0}$ has character values in $F$. Since all the character values are in $K$ by the previous paragraph, it suffices to show that all character values are real.

Since $\tau$ inverts all conjugacy classes in $B_{0} S_{m}$, we need only show that if $g=b s \tau$ with $b \in B_{0}$ and $s \in S_{m}$, then $g$ and $g^{-1}$ are conjugate in $G_{0}$. We prove the formally stronger statement allowing $b \in B$ rather and show $g$ is conjugate to $g^{-1}$ via an element in $G_{0}$.

By induction on $m$, it suffices to prove this result when $s$ is an $m$-cycle. Note that we can replace $g$ by any $G$-conjugate.

There is no harm in assuming that $s$ is the $m$-cycle $(12 \ldots m)$. By conjugating by an element of $B$, we may assume that $b=\left(a_{1}, \ldots, a_{m}\right)$ where $a_{i} \in A^{2}$ for $i>1$. If $a_{1} \in A^{2}$, then $g \in\left(A^{2}\right)^{m}\left(S_{m} \times\langle\tau\rangle\right.$, and all representations of this group are defined over $K$ by Lemma 6.1. If $a_{1}$ is not a square in $A$, then $g$ is not in $B_{0}$. Since $g^{x}=g^{-1}$ for some $x \in G$, one of $x$ and $g x$ is in $G_{0}$ and conjugates $g$ to $g^{-1}$.

\section{Classical Weyl groups}

Let $G$ be a classical Weyl group. We prove Theorem 1.1 and (1) of Theorem 1.4 for the classical Weyl groups in this section. By Corollary 4.5] it suffices to work over $\mathbb{C}$.

We have already shown that all Schur indicators for $C_{g}$ are +1 when $g^{2}=1$ (and all irreducible modules are defined over the prime field).

Now consider $g^{2} \neq 1$. We have already shown that if $x \in N_{g} \backslash C_{g}$, then $x$ inverts all conjugacy classes of $C_{g}$. So all that remains to prove is that all Schur indicators are +1 for $N_{g}$ when $g^{2} \neq 1$.

We first handle the case of $\operatorname{Sym}_{n}$. We prove a slightly more general result. Namely, let $J$ be an abelian subgroup of $\operatorname{Sym}_{n}$ with centralizer $C$. Let $N=\langle C, \tau\rangle$, where $\tau$ acts as inversion on $C$. If $J$ is elementary abelian, then we have shown that all representations of $C=N$ are defined over $\mathbb{Q}$. So assume that $J$ has an exponent greater than 2. If $J$ has an orbit that is not regular, then $C=C_{1} \times \ldots \times C_{t}$ where $C_{i}$ is the centralizer of $J$ acting on $\Omega_{i}$ and $\Omega_{i}$ is the union of the $J$-orbits with a given point stabilizer. Applying Lemma 3.4 yields the result by induction. So we may assume that all orbits of $J$ are regular. Then $N$ is the group described in Lemma 6.1 and we have seen that all representations are defined over $\mathbb{R}$.

The case where $G$ is of type $B_{n}$ now follows (for the more general case of abelian subgroups $J$ containing $Z(G)$ ) since $C$ and $N$ will be contained in $G \leq \operatorname{Sym}_{2 n}$.

Finally, consider the case that $G=D_{n}$. By Lemma 3.2 and the result for $B_{n}$, it suffices to prove that all irreducible representations of $N_{g}$ are self dual. It follows by Theorem 1.2 that $y^{x}$ is conjugate to $y^{-1}$ in $N_{g}$ for any $y \in C_{g}$ and any $x \in N_{g} \backslash C_{g}$. 
So it suffices to show:

Lemma 7.1. Let $W$ be a Weyl group of types $A, B$ or D. Let $g \in W$ and $h \in N_{g} \backslash C_{g}$. Then there exists $x \in N_{g}$ inverting $h$.

Proof. In fact, since we have proved that all representations of $N_{g}$ have a Schur indicator +1 for $W$ of type $\mathrm{A}$ and $\mathrm{B}$, the result holds in that case. We prove a slightly stronger result for type $\mathrm{B}$ that will give the result for type $\mathrm{D}$ as well.

Let $W=B_{n}$. Let $W_{0}$ be the subgroup of index 2 of type $\mathrm{D}$ in $W$. We prove the slightly stronger result that given $h \in N_{g} \backslash C_{g}$, there exists $x \in W_{0} \cap N_{g}$. There is also no harm in assuming that $g$ has even order $r$ (for if $g$ has odd order, replace $g$ by $g z$, where $z$ is the central involution of $W$ ).

Since all representations of $N_{g}$ are defined over $\mathbb{R}$, it follows that there is some $x \in N_{g}$ inverting $h$ (indeed, by replacing $x$ by $x h$, we can choose $x \in C_{g}$ ). We need to show that we can choose $x \in C_{g} \cap W_{0}$.

We induct on $n$. The case $n \leq 3$ is clear. Set $X=\langle g, h\rangle$.

If $C_{W}(X)$ is not contained in $W_{0}$, the result follows as well (for choose $w$ in $C_{W}(X)$ with $w$ not in $W_{0}$; then either $x \in W_{0}$ or $x w \in W_{0}$, and both elements invert $h)$.

In particular, if $n$ is odd, the result follows, for then $Z(W)$ is not contained in $W_{0}$.

We view $W \leq \mathrm{Sym}_{2 n}$ centralizing the fixed point free involution $z$. Note that if $X$ is contained in a direct product of subgroups of $W$ of type $\mathrm{B}$, the result holds by induction. In particular, this implies that we may assume that $g$ is homogeneous i.e. viewing $W \leq \operatorname{Sym}_{2 n}, g$ has all orbits of size $r=2 s$. The number of orbits is $m:=n / s$. Similarly, $\langle g, z\rangle$ has all orbits of the same size $r^{\prime}$. So either $z$ is a power of $g$ and $r^{\prime}=r$ or $r^{\prime}=2 r$.

If $C_{W}(g) \leq W_{0}$, then as $g^{y}=g^{-1}$ for some $y \in W_{0}$ (since all representations of $W_{0}$ are real), $N_{g} \leq W_{0}$ and $x \in N_{g} \leq W_{0}$. This is the case if $r^{\prime}=2 r$. So we may assume that $r^{\prime}=r$, i.e. $z$ is a power of $g$.

In this case, $C_{g} \cong \mathbb{Z} / 2 s \imath S_{m}$. It follows that $N_{g}=A^{m} \rtimes\left(S_{m} \times\langle\tau\rangle\right)$, where $A$ is cyclic of order $2 m$ and $\tau$ inverts $A^{t}$. By Lemma 6.2, $N_{g}$ is a real group, and so the conclusion holds.

\section{General Real reflection Groups}

We have now shown that if $W$ is a Weyl group of classical type and $g \in W$, then:

(1) $N_{g}$ is totally orthogonal for every $g \in W$.

(2) Every element of $C_{g}$ is inverted by an element of $N_{g} \backslash C_{g}$.

(3) $D(W)$ is totally orthogonal.

It is obvious that all three properties hold for dihedral groups as well. The remaining real reflection groups are $H_{3}, H_{4}$ and the Weyl groups of type $F_{4}, E_{6}$, $E_{7}$ and $E_{8}$ (see [1] ). Since $H_{3}=A_{5} \times \mathbb{Z} / 2$, it is clear that all three conditions hold for $\mathrm{H}_{3}$ as well.

For the remaining 5 groups, we compute using MAGMA or GAP. All the character tables of these groups are in MAGMA (or GAP), and it is straightforward to loop through the irreducible modules $C_{g}$ and $N_{g}$ and compute their indicators. We see that over $\mathbb{C}, C_{g}$ is totally orthogonal for $g^{2}=1$ and $\nu(V) \geq 0$ for all irreducible $\mathbb{C} N_{g}$-modules $V$. 
Using Theorem 2.6 and the character tables for $N_{g}$, we see that $\nu(\hat{V})=+1$ for irreducible $D(G)$-modules (over $\mathbb{C}$ ). By the reduction lemma, this holds for any odd characteristic as well.

We also note for $H_{4}$ and the Weyl groups of type $E$ and $F$ that $N_{g}$ need not be totally orthogonal. Indeed, in each case, there is an element $g$ of order 3 such that some irreducible $\mathbb{C} N_{g}$-module has Schur indicator 0 (but all indicators for $\mathbb{C} N_{g}$ are non-negative).

This suggests that the method of proof used for the classical Weyl groups will not extend to all Weyl groups. The example in the next section also shows that even being split over $\mathbb{Q}$ does not imply that $D(G)$ cannot have irreducible modules with indicators 0 .

We end this section by proving a result which includes Corollary 1.5

Theorem 8.1. Let $G$ be a finite group with Drinfel'd double $D(G)$ (over $\mathbb{C}$ ). Let $t$ be the number of elements $g \in G$ with $g^{2}=1$. Then

$$
t^{2} \leq \sum_{g \in G} \sum_{\chi \in \operatorname{Irr}\left(C_{g}\right)} \chi(1)
$$

with equality if and only if $D(G)$ is totally orthogonal.

Proof. Let $S$ be the antipode of $D(G)$. Then it follows from 18 that the trace of $S$ on $D(G)$ is the number of pairs $(a, g)$ with $a^{2}=1=(a g)^{2}$. It is easy to see that this is $t^{2}$.

It also follows from [18] that this trace is $\sum \nu(W) \operatorname{dim} W$, where the sum is over all distinct irreducible $D(G)$-modules. Let $\Omega$ be a set of representatives of conjugacy classes of $G$.

It follows from the description of the irreducible $D(G)$-modules; this is

$$
\sum_{g \in \Omega}\left[G: C_{g}\right] \sum_{V \in \operatorname{Irr}\left(C_{g}\right)} \operatorname{dim} V=\sum_{g \in G} \sum_{V \in \operatorname{Irr}\left(C_{g}\right)} \operatorname{dim} V .
$$

Thus, the inequality holds with equality precisely when $\nu(W)=+1$ for irreducible $D(G)$-modules.

See 12 for a generalization of this result.

\section{An EXAmple}

We now give an example to show that one cannot extend the main theorem to groups in which all representations are defined over $\mathbb{R}$ (or even $\mathbb{Q}$ ).

Let $G=\left\langle x, a, b \mid x^{8}=a^{2}=b^{2}=1, a x a=x^{-1}, b x b=x^{3}\right\rangle$.

Note that $G$ has order 32 and is the holomorph of the cyclic group of order 8 (i.e. it is the semidirect product of the cyclic group of order 8 and its automorphism group).

Lemma 9.1. All representations of $G$ are defined over $\mathbb{Q}$.

Proof. Note that $x^{4}$ is central in $G$ and $G /\left\langle x^{4}\right\rangle \cong D_{8} \times \mathbb{Z} / 2$, where $D_{8}$ is the dihedral group of order 8. All representations of $D_{8}$ are easily seen to be defined over $\mathbb{Q}$, hence also those representations of $G$ with $x^{4}$ acting trivially.

We now construct a faithful 4-dimensional absolutely irreducible representation of $G$ defined over $\mathbb{Q}$. Namely, let $V=\mathbb{Q}\left(\zeta_{8}\right)$, the field generated by the 8th roots of unity over $\mathbb{Q}$. This is a 4 -dimensional module over $\mathbb{Q} ; G$ acts on it by letting $x$ act 
by multiplication by $\zeta_{8}$ and $\langle a, b\rangle$ by $\operatorname{Gal}(V)$. It is easy to see that these actions give a group isomorphic to $G$.

Now $x$ has 4 distinct eigenvalues in this representation (the primitive 8 th roots of 1) and $G$ permutes these transitively. Any submodule must be generated by $\langle x\rangle$-eigenspaces, and therefore is either 0 or contains all the eigenspaces. Thus $V$ is an (absolutely) irreducible 4-dimensional module defined over $\mathbb{Q}$.

Adding the sum of the squares of the degrees, we see that this gives all modules and all are defined over $\mathbb{Q}$.

Lemma 9.2. Let $g=x a$. Then $g^{2}=1$ and $C_{G}(g)=\left\langle x^{2} a b, g\right\rangle \cong \mathbb{Z} / 4 \times \mathbb{Z} / 2$.

Proof. It is straightforward to compute that $g$ is an involution and commutes with $x^{2} a b$, an element of order 4. Since $C_{G}(g) \cap\langle x\rangle=\left\langle x^{4}\right\rangle$, it follows that $\left|C_{G}(g)\right| \leq 8$. Since $C_{G}(g) \geq\left\langle x^{2} a b, g\right\rangle$, we have equality.

In particular, we see that for the involution $g, C_{g}$ has two non-self dual representations (this can also be seen using MAGMA). This implies that the Schur indicators for the corresponding modules for $D(G)$ have Schur indicator 0 as well.

So $G$ has all indicators +1 , but $D(G)$ has some indicators 0 .

Question. Can there be negative indicators for $D(G)$ if all representations of $G$ are defined over $\mathbb{R}$ or $\mathbb{Q}$ ?

See [8, 9, 30] for examples of totally orthogonal groups.

\section{REFERENCES}

[1] P. Bantay, The Frobenius-Schur indicator in conformal field theory, Physics Lett. B 394 (1997), no. 1-2, 87-88. MR.1436801 (98c:81195)

[2] P. Bantay, Frobenius-Schur indicators, the Klein-bottle amplitude, and the principle of orbifold covariance, Phys. Lett. B 488 (2000), 207-210. MR.1782167 (2001e:81094)

[3] T. Breuer, in preparation.

[4] R. Dijkgraaf, V. Pasquier, P. Roche, QuasiHopf algebras, group cohomology and orbifold models, Nucl. Phys. B Proc. Suppl. 18B (1990), 60-72. MR.1128130 (92m:81238)

[5] W. Feit, The representation theory of finite groups, North-Holland Mathematical Library, 25. North-Holland Publishing Co., Amsterdam-New York, 1982. MR661045 (83g:20001)

[6] J. Fuchs, A. Ch. Ganchev, K. Szlachányi and P. Vecsernyés, $S_{4}$ symmetry of $6 j$ symbols and Frobenius-Schur indicators in rigid monoidal $C^{*}$ categories. J. Math. Phys. 40 (1999), no. 1, 408-426. MR:1657800 (99k:81111)

[7] D. Goldstein and R. Guralnick, Rationality of certain subgroups of Weyl groups, preprint.

[8] R. Gow, Real representations of the finite orthogonal and symplectic groups of odd characteristic, J. Algebra 96 (1985), 249-274. MR808851 (87b:20015)

[9] R. Gow, Properties of the characters of the finite general linear group related to the transposeinverse involution, Proc. London Math. Soc. (3) 47 (1983), 493-506. MR716800 (85k:20130)

[10] L. C. Grove and K. S. Wang, Realizability of representations of finite groups, J. Pure Appl. Algebra 54 (1988) 299-310. MR.963550 (89g:20018)

[11] J. Humphries, Reflection groups and Coxeter groups, Cambridge Studies in Advanced Mathematics 29, Cambridge University Press, 1990. MR1066460 (92h:20002)

[12] A. Jedwab and S. Montgomery, Representations of some Hopf algebras associated to the symmetric groups, preprint.

[13] Y. Kashina, On semisimple Hopf algebras of dimension $2^{m}$, Algebras and Representation Theory 6 (2003), no. 4, 393-425. MR2020263 (2005f:16058)

[14] Y. Kashina, G. Mason, and S. Montgomery, Computing the Frobenius-Schur indicator for abelian extensions of Hopf algebras, J. Algebra 251 (2002), 888-913. MR1919158 (2003f:16061)

[15] Y. Kashina, Y. Sommerhäuser, and Y. Zhu, Self-dual modules of semisimple Hopf algebras, J. Algebra 257 (2002), 88-96. MR1942273(2003m:16052) 
[16] Y. Kashina, Y. Sommerhäuser, and Y. Zhu, On higher Frobenius-Schur indicators, AMS Memoirs 181 (2006), no. 855, 65 pp. MR2213320(2007k:16071)

[17] R. Larson and D. Radford, Semisimple cosemisimple Hopf algebras, Amer. J. Math. 110 (1988), 187-195. MR926744 (89a:16011)

[18] V. Linchenko and S. Montgomery, A Frobenius-Schur theorem for Hopf algebras, Algebras and Representation Theory, 3 (2000), 347-355. MR.1808131(2001k:16073)

[19] G. Mason, The quantum double of a finite group and its role in conformal field theory, Groups '93 Galway/St. Andrews, Vol. 2, 405-417, London Math. Soc. Lecture Note Ser., 212, Cambridge Univ. Press, Cambridge, 1995. MR1337285 (97a:11067)

[20] G. Mason and S-H. Ng, Central invariants and Frobenius-Schur indicators for semisimple quasi-Hopf algebras, Advances in Math. 190 (2005), 161-195. MR2104908 (2005h:16066)

[21] A. Masuoka, Faithfully flat forms and cohomology of Hopf algebra extensions, Comm. Algebra 25 (1997), 1169-1197. MR1437667 (98b:16034)

[22] S. Montgomery, Representation theory of semisimple Hopf algebras, Algebra - Representation Theory (Constanta, 2000), K. W. Roggenkamp and M. Stefanescu, editors, NATO Sci. Ser. II Math. Phys. Chem. 28, Kluwer, Dordrecht, 2001, 189-218. MR.1858037 (2002g:16062)

[23] S-H. Ng and P. Schauenburg, Central invariants and higher indicators for semisimple quasiHopf algebras, Trans. Amer. Math. Soc. 360 (2008), no. 4, 1839-1860. MR2366965

[24] S-H. Ng and P. Schauenburg, Frobenius-Schur indicators and exponents of spherical categories, Advances in Math. 211 (2007), 34-71. MR2313527 (2008b:16067)

[25] S-H. Ng and P. Schauenburg, Higher Frobenius-Schur indicators for pivotal categories, AMS Contemporary Math., to appear.

[26] S. Natale, Frobenius-Schur indicators for a class of fusion categories, Pacific J. Math., 221 (2005), no. 2, 353-377. MR2196640 (2007j:16070)

[27] P. Schauenburg, On the Frobenius-Schur indicators for quasi-Hopf algebras, J. Algebra 282 (2004), 129-139. MR2095575 (2005h:16068)

[28] T. Springer, Regular elements of finite reflection groups, Invent. Math. 25 (1974), 159-198. MR0354894 (50:7371)

[29] J. G. Thompson, Bilinear forms in characteristic $p$ and the Frobenius-Schur indicator, Lecture Notes Math. 1185 (1986), pp. 221-230.

[30] P. H. Tiep and A. Zalesski, Real conjugacy classes in algebraic groups and finite groups of Lie type, J. Group Theory, 8 (2005), 291-315. MR2137972(2006b:20010)

[31] S. Witherspoon, The representation ring of the quantum double of a finite group, J. Algebra 179 (1996), 305-329. MR1367852 (96m:20015)

Department of Mathematics, University of Southern California, Los Angeles, CalIFORNIA 90089-2532

E-mail address: guralnic@usc.edu

Department of Mathematics, University of Southern California, Los Angeles, CalIFORNIA 90089-2532

E-mail address: smontgom@usc.edu 